\title{
Influence of selected modeling and computational issues on muscle force estimates
}

\author{
Wojciech Blajer • Adam Czaplicki • \\ Krzysztof Dziewiecki · Zenon Mazur
}

Received: 6 October 2009 / Accepted: 1 July 2010 / Published online: 1 August 2010

(C) The Author(s) 2010. This article is published with open access at Springerlink.com

\begin{abstract}
Knowledge of muscle forces and joint reaction forces during human movement can provide insight into the underlying control and tissue loading. Since direct measurement of the internal loads is generally not feasible, non-invasive methods based on musculoskeletal modeling and computer simulations have been extensively developed. By applying observed motion data to the musculoskeletal models, inverse dynamic analysis allow to determine the resultant joint torques, transformed then into estimates of individual muscle forces by means of different optimization procedures. Assessment of the joint reaction forces and other internal loads is further possible. Comparison of the muscle force estimates obtained for different modeling assumptions and parameters in the model can be valuable for the improvement of validity of the model-based estimations. The present study is another contribution to this field. Using a sagittal plane model of an upper limb with a weight carried in hand, and applying the data of recorded flexion and extension movement of the upper limb, the resultant muscular forces are predicted using different modeling assumptions and simulation tools. This study relates to different coordinates (joint and natural coordinates) used to built the mathematical model, muscle path modeling, muscle decomposition (change in number of the modeled muscles), and different optimization methods used to share the joint torques into individual muscles.
\end{abstract}

\footnotetext{
W. Blajer $(\bowtie) \cdot$ K. Dziewiecki · Z. Mazur

Faculty of Mechanical Engineering, Technical University of Radom, ul. Krasickiego 54, 26-600 Radom, Poland

e-mail: w.blajer@pr.radom.pl

K. Dziewiecki

e-mail: krzysztof.dziewiecki@pr.radom.pl

Z. Mazur

e-mail: z.mazur@pr.radom.pl
}

\section{A. Czaplicki}

External Faculty of Physical Education in Biała Podlaska, Department of Biomechanics, The Academy of Physical Education in Warsaw, ul. Akademicka 2, 21-500 Biała Podlaska, Poland

e-mail: czaplicki@poczta.onet.pl 
Keywords Musculoskeletal system modeling · Inverse dynamics · Muscle force sharing problem · Optimization

\section{Introduction}

Knowledge of muscle forces and joint reaction forces during human movement can provide insight into the underlying neural control and tissue loading, and can thus be of great importance for the design of prostheses, development of post-operative regimes, and assessment of risk of damage of ligaments during various sport activities. Since direct measurement of the internal loads is generally not feasible, non-invasive methods based on musculoskeletal modeling and computer simulations have been extensively developed [1-3].

The inverse dynamics technique applied to musculoskeletal models is used extensively to calculate the muscular joint torques required to generate an observed motion. Since this gives only a very general information about intensity of the required actuation, the muscular loads are then shared, by means of static optimization procedures [4-7], into the individual muscles whose tensile forces acting on the bones (via tendons) exert moments about the joints of the skeletal system. Assessment of internal loads within the joints, influenced by the muscle forces, is further possible. Irrespective of the lack of studies reporting successful experimental validation of the model-based estimates of muscle forces, a continuing interest and substantial progress in the development of musculoskeletal systems modeling, identification and simulation is observed [5, 6, 8-12], devoted to improving accuracy of the solutions.

The muscle force estimates resulted from the inverse dynamics-based static optimization are sensitive to various issues [6]. Several investigations studied the sensitivity of the results to anthropometrical parameters used in the human models. Important is accurate identification of the origin and insertion points $[13,14]$ and the muscle paths relative to the skeleton $[13,15,16]$, which determine the muscle moment arms around the joints, and as such, increase/decrease the muscle force estimates. The magnitudes of muscle force estimates are also sensitive to the muscle maximum isometric stresses and physiological cross-sectional areas [17]. Inclusion of a model of muscle-tendon dynamics can also have significant effects on the results [10, 17-19]. Multiple objective functions applied in the optimization have also been proposed and tested with respect to muscle force sharing problem [4]. Finally, accuracy of the experimental data used within the model is proven to be of paramount importance for the accurate muscle force estimations [20]. A thorough discussion on the mentioned aspects of model-based estimation of muscle forces exerted during movements, with a wide review of the literature in the field, is provided in [6].

Comparison of the muscle force estimates obtained for different modeling assumptions, different objective functions used in the optimization methods, and slightly altered parameters and/or experimental data used in the models can be valuable for the improvement of validity of the model-based estimations. The present study is another contribution to this field. Using a sagittal plane model of an upper limb with a weight carried in hand, and applying the data of recorded flexion and extension movement of the upper limb, the resultant muscular forces (and joint reaction forces) are predicted using different modeling assumptions and simulation tools, and the results are compared to each other. Two different mathematical models of the limb are tested, derived, respectively in joint and natural coordinates. Influence of modeling of musculotendon paths near the joints is then examined, the problem raised previously in, e.g. $[13,15,16]$. The comparative study include also influence of muscle decomposition (change in number of the modeled muscles), and different optimization methods used to share the joint torques into the individual muscles. 


\section{The upper limb model}

The upper limb musculoskeletal model used in this study is seen in Fig. 1. It is designed as a planar kinematic structure composed of the arm (segment S1) and forearm together with a weight carried in hand (treated as one segment $S 2$ ) connected by the elbow (ideal hinge) joint $E$, and then attached to the trunk (segment $S O$ ) through the shoulder (another ideal hinge) joint $S$. The $S$ joint follows a specified in time (measured) motion $\mathbf{r}_{S d}(t)=$ $\left[\begin{array}{lll}x_{S d}(t) & y_{S d}(t)\end{array}\right]^{T}$, and the $n=2$ coordinates that describe the upper limb position are $\mathbf{q}=$ $\left[\begin{array}{ll}\varphi_{1} & \varphi_{2}\end{array}\right]^{T}$, where $\varphi_{1}$ and $\varphi_{2}$ are angles that measure deviations of the segments from the vertical downward positions. The multibody system is actuated by $k=8$ muscles, numbered from $m l$ to $m 8$ in Fig. 1, and as such the system in overactuated, $k>n$.

It was long since it had been documented that assumptions used to define the musculotendon paths (and consequently moment arms) near the joints are of critical importance for the musculoskeletal model behavior and reliability of the consequent muscle force estimates $[13,15,16]$. In many applications, the musculotendon paths are defined as the lines connecting the actual origin and insertion points of the muscles. Such practice is neither physiologically grounded nor mechanically correct, however. The mechanical consequence is that, in some particular configurations, the muscle force arms with respect to the joints may be very small or even vanishing. These effects make estimation of muscle forces ill posed or at least unreliable at these specific configurations. The situation is illustrated in Fig. 2 for muscles $m 5$ (attached to $S O$ and $S 2$ segments) and $m 6$ (attached to $S 1$ and $S 2$ segments). While in configuration seen in Fig. 2a, the muscles can exert moment about the elbow joint $E$, and as such can contribute to the torque required to accelerate extension (or decelerate flexion) movements in the joint, in configuration seen in Fig. 2b, this would require very large/infinite muscle forces due to the fact that their moment arms with respect to the joint are vanishing.

The effective musculotendon path model is defined by assuming effective origin and/or insertion points and drawing a straight line between these points. Implicit in this model is that, in the neighborhood of the joint, the muscle path arcs around a cylindrical (spherical in 3D models) shell so that a certain moment arm is reached irrespective to the relative configuration of segments of the musculoskeletal model. The effective path model for muscles $m 5$ and $m 6$ crossing the elbow joint $E$ is illustrated in Fig. 3. Now, the muscles can exert moment about the joint in the whole range of the joint angle. Evidently, the muscle path

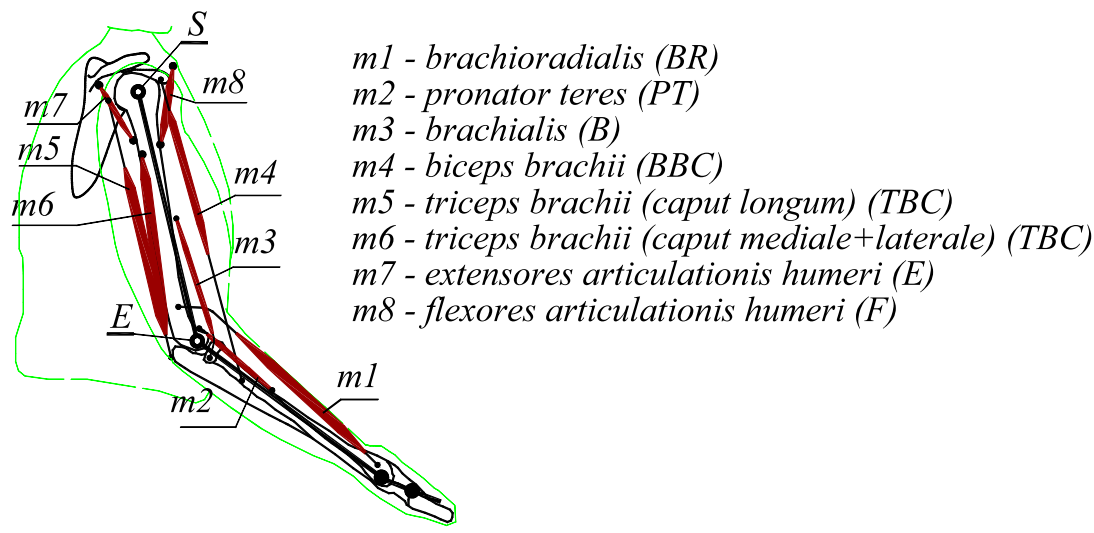

Fig. 1 The upper limb musculoskeletal model 
Fig. 2 The origin-insertion model of muscle path a)
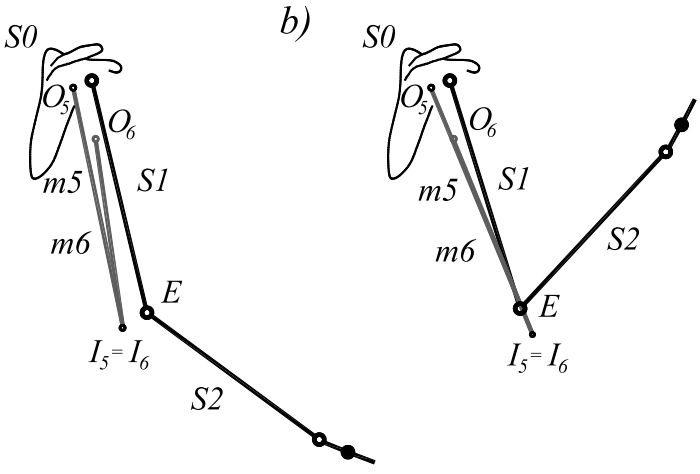

Fig. 3 The effective muscle path model

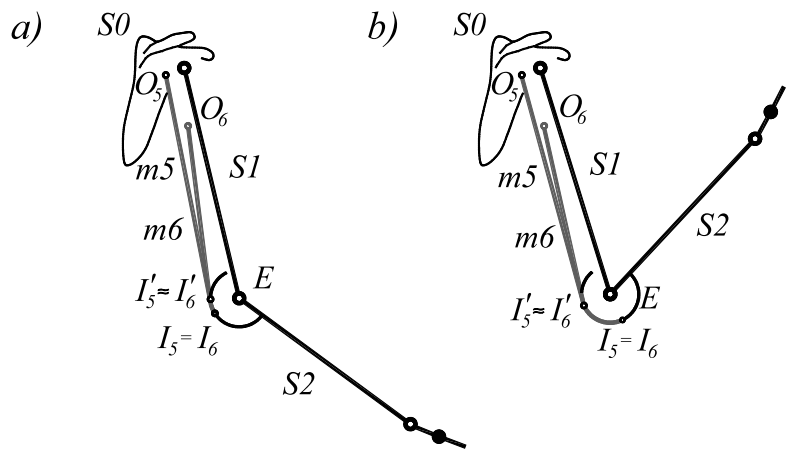

model influences the effective muscle length, and the effective origin/insertion points need to be specified (and redefined) for each configuration. There is always some modeling effort concerned with these 'geometrical' issues, which will not be reported in this contribution for shortness.

The muscle moment arms, derived from musculoskeletal anatomy, may or may not depend on joint angles. In the upper limb model used in this study, reassumed in Fig. 4, constant and equal values of radiuses defining the force arms of muscles $m 4, m 5, m 7$, and $m 8$ with respect to the shoulder joint $S$ were assumed, $r_{S 4}=r_{S 5}=r_{S 7}=r_{S 8}=25 \mathrm{~mm}$. The radiuses $r_{E j}$ of muscles $m 1, m 2, m 3, m 4, m 5$, and $m 6$ that exert torques about the elbow joint $E, j=1, \ldots, 6$, were taken after [21-23] as dependent on the relative angle $\alpha$ between the arm and forearm, which is represented in Fig. 5. In calculations, the characteristics were used in the form of cubic polynomials

$$
r_{E j}(\alpha)=a_{j} \alpha^{3}+b_{j} \alpha^{2}+c_{j} \alpha+d_{j}
$$

and the coefficients $a_{j}, b_{j}, c_{j}$, and $d_{j}$ are reported in Table 1 .

The physiological cross-sectional areas $A_{j}$ (abbreviated hereafter from $P C S A_{j}$ ) used in calculations for the muscles $m 1, \ldots, m 8$ are seen in Table 1 . Then we assumed the same physiological allowable minimal and maximal values of muscle stresses for all the muscles, respectively, $\sigma_{\min }=0.01 \mathrm{MPa}$ and $\sigma_{\max }=0.75 \mathrm{MPa}$, which determine the allowable minimal and maximal values of muscle forces, $F_{j \min }=A_{j} \sigma_{\min }$ and $F_{j \max }=A_{j} \sigma_{\max }$. Finally, in Table 2, the geometric and inertial parameters of segments $S 1$ and $S 2$ are represented. 

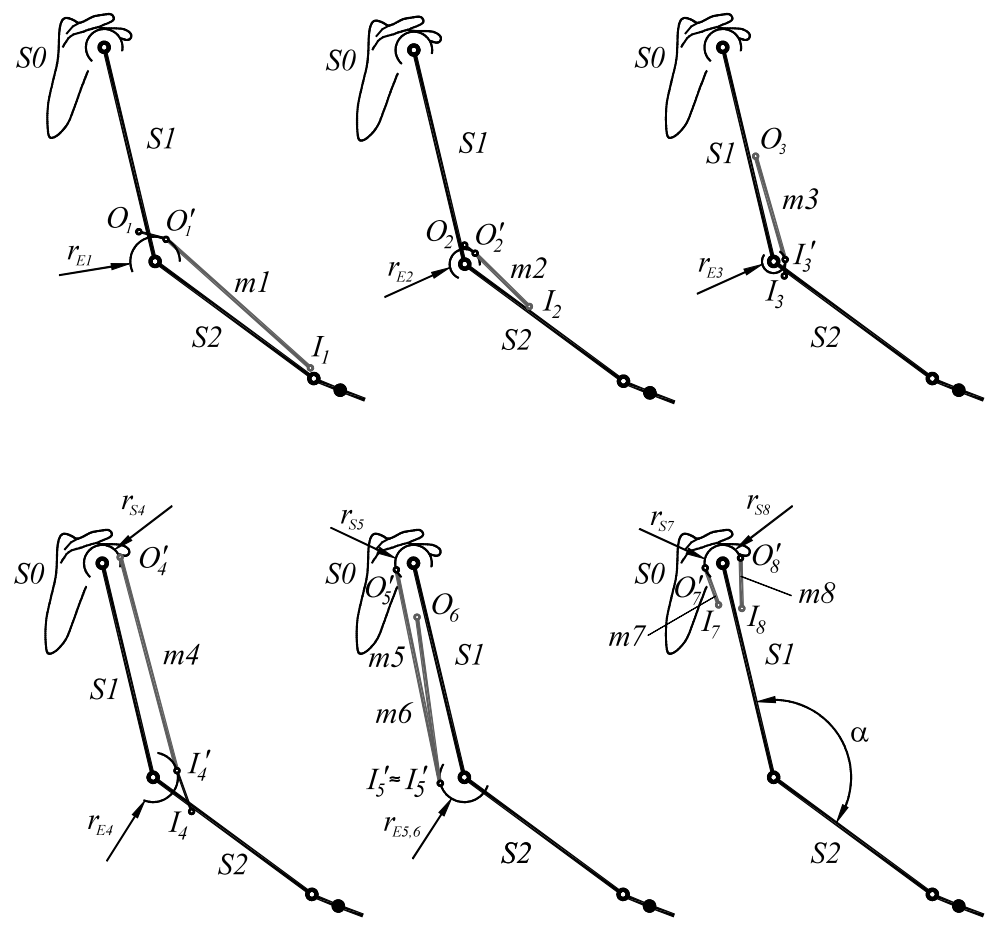

Fig. 4 The effective musculotendon paths of the upper limb model

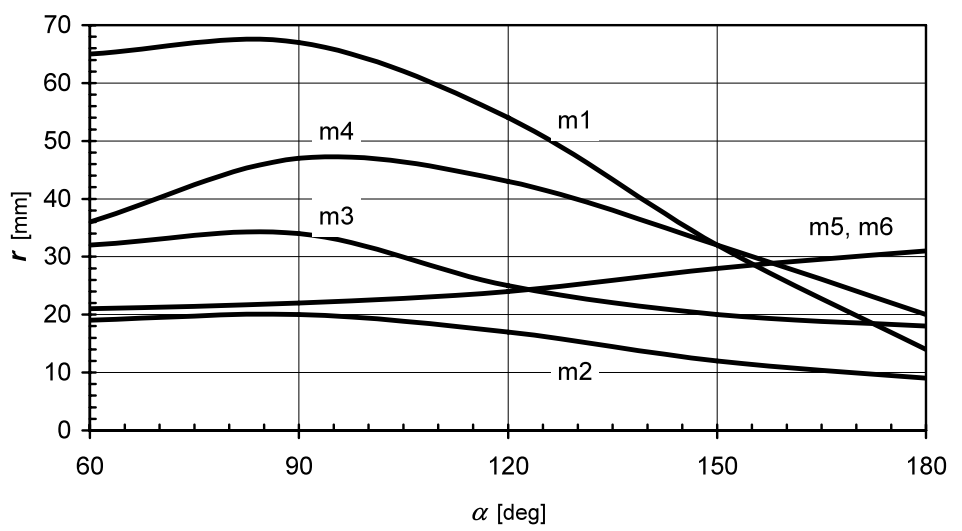

Fig. 5 The muscle force arms in the elbow joint

\section{Mathematical modeling}

For comparison reasons of this study, two qualitatively different mathematical models of the upper extremity were built, which introduce the dynamic equations derived in terms of, respectively, joint coordinates and natural coordinates. While the first coordinate type is commonly used in biomechanical system modeling [1-3, 24-26], implementations of the other coordinates in biomechanics are rather rare [5, 12, 27-29]. By comparing the 
Table 1 Effective path musculotendon parameters used in the model

\begin{tabular}{lrlllr}
\hline Muscle & $\begin{array}{l}A \\
\mathrm{~mm}^{2}\end{array}$ & $\begin{array}{l}a \\
\mathrm{~mm} / \mathrm{deg}^{3}\end{array}$ & $\begin{array}{l}b \\
\mathrm{~mm} / \mathrm{deg}^{2}\end{array}$ & $\begin{array}{l}c \\
\mathrm{~mm} / \mathrm{deg}\end{array}$ & $\begin{array}{l}d \\
\mathrm{~mm}\end{array}$ \\
\hline$m 1:$ BR & 150 & $5.864 \mathrm{E}-05$ & $-2.500 \mathrm{E}-02$ & $2.831 \mathrm{E}+00$ & $-2.760 \mathrm{E}+01$ \\
$m 2:$ PT & 300 & $1.852 \mathrm{E}-05$ & $-7.460 \mathrm{E}-03$ & $8.405 \mathrm{E}-01$ & $-8.600 \mathrm{E}+00$ \\
$m 3:$ B & 500 & $4.321 \mathrm{E}-05$ & $-1.587 \mathrm{E}-02$ & $1.671 \mathrm{E}+00$ & $-2.020 \mathrm{E}+01$ \\
$m 4:$ BBC & 750 & $4.321 \mathrm{E}-05$ & $-1.976 \mathrm{E}-02$ & $2.587 \mathrm{E}+00$ & $-5.740 \mathrm{E}+01$ \\
$m 5:$ TBC c.lon. & 500 & $-6.173 \mathrm{E}-06$ & $2.698 \mathrm{E}-03$ & $-2.754 \mathrm{E}-01$ & $2.920 \mathrm{E}+01$ \\
$m 6:$ TBC c.lat.med. & 900 & $-6.173 \mathrm{E}-06$ & $2.698 \mathrm{E}-03$ & $-2.754 \mathrm{E}-01$ & $2.920 \mathrm{E}+01$ \\
$m 7:$ E & 1500 & - & - & - & - \\
$m 8:$ F & 1500 & - & - & & - \\
\hline
\end{tabular}

Table 2 Mechanical parameters of the model segments

\begin{tabular}{llllll}
\hline Segment & $m$ & $l$ & $\xi_{C}$ & $\eta_{C}$ & $J_{C}$ \\
& $\mathrm{~kg}$ & $\mathrm{~m}$ & $\mathrm{~m}$ & $\mathrm{~kg} \mathrm{~m}^{2}$ \\
\hline$S 1$ & 3.2 & 0.311 & 0.184 & -0.016 & 0.035 \\
$S 2$ & 5.2 & 0.295 & 0.256 & -0.005 & 0.022 \\
\hline
\end{tabular}

simulation results obtained using these two mathematical models, one can easily eliminate possible defects in the models. Apart from this aim, the virtual objective of this study was to verify a supposition that the optimization results may depend on the choice of type/number of variables used to describe configuration of biomechanical models, which influence the structure and number of the dynamic equations that arise.

The general schemes for deriving the dynamic equations of biomechanical systems, especially for planar structures such as that studied in this paper, are evidently not new and well documented in the literature. The present formulations for the upper limb planar model were built using the codes described previously in [26] and [29], when using, respectively, the joint and natural coordinates. Therefore, in the following, we recall only the basic ideas of the methods, which are necessary for completeness of this presentation, and concentrate more on the details related to the upper limb model at hand.

\subsection{Dynamic equations in joint coordinates}

The dynamic equations of the upper extremity upon study, in terms of $n=2$ joint coordinates $\mathbf{q}=\left[\begin{array}{ll}\varphi_{1} & \varphi_{2}\end{array}\right]^{T}$ (Fig. 6a), can conveniently be derived using the projective scheme described in [26]. Following this procedure, the starting point are the dynamic equations in $N=3 n=6$ absolute coordinates $\mathbf{p}=\left[\begin{array}{llllll}x_{C 1} & y_{C 1} & \theta_{1} & x_{C 2} & y_{C 2} & \theta_{2}\end{array}\right]^{T}$, where $x_{C i}, y_{C i}$ are the coordinates of the mass centers $C_{i}$ in the inertial frame $X Y$, and $\theta_{i}$ are the orientation angles (here $\theta_{i}=\varphi_{i}$ ) of upper extremity segments $S 1$ and $S 2$ (Fig. 6b). More strictly, from the dynamic equations in $\mathbf{p}$, which can generally be written as

$$
\mathbf{M} \ddot{\mathbf{p}}=\mathbf{f}_{g}-\mathbf{C}^{T} \lambda+\mathbf{B} \boldsymbol{\sigma}
$$

one needs to introduce explicitly only $\mathbf{M}, \mathbf{f}_{g}$, and $\mathbf{B}$, where $\mathbf{M}=\operatorname{diag}\left(m_{1}, m_{1}, J_{C 1}, m_{2}\right.$, $\left.m_{2}, J_{C 2}\right)$ is the generalized mass matrix related to $\mathbf{p}, m_{i}$, and $J_{C i}$ are the mass and mass moment of inertia with respect to $C_{i}$ of the segments, $\mathbf{f}_{g}=\left[\begin{array}{llllll}0 & -m_{1} g & 0 & 0 & -m_{2} g & 0\end{array}\right]^{T}$ 
a)

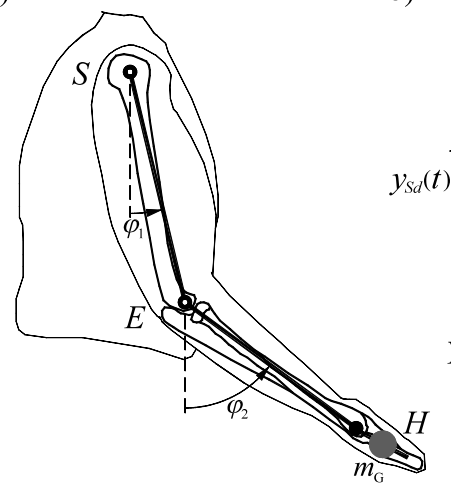

b)

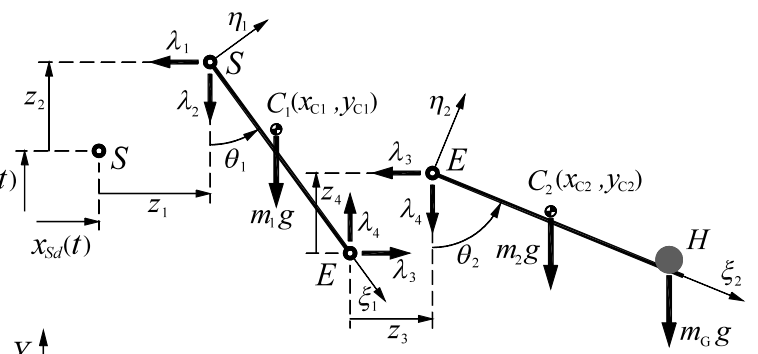

Fig. 6 The joint and absolute coordinates

contains the gravitational forces, $\mathbf{f}_{\sigma}=\mathbf{B} \sigma$ is the $N$-vector of generalized control force, and $\mathbf{B}(\mathbf{p})$ is the $N \times m$-dimensional matrix of distribution of $m=8$ control inputs $\sigma=$ $\left[\begin{array}{lll}\sigma_{1} & \cdots & \sigma_{8}\end{array}\right]^{T}$, where $\sigma_{j}=F_{j} / A_{j}$ are the muscle stresses, $j=1, \ldots, 8$. Then in (2), $\mathbf{f}_{\lambda}=-\mathbf{C}^{T} \lambda$ is the $N$-vector of generalized reaction force due to $l=4$ constraints on the segments, $\mathbf{z}=\boldsymbol{\Phi}(\mathbf{p})=\mathbf{0}$, where $\mathbf{C}(\mathbf{p})=\partial \boldsymbol{\Phi} / \partial \mathbf{p}$ is the $l \times N(4 \times 6)$ constraint matrix, and $\lambda=\left[\begin{array}{llll}\lambda_{1} & \lambda_{2} & \lambda_{3} & \lambda_{4}\end{array}\right]^{T}$ contains the constraint reaction forces. Neither expanded forms of constraints $\boldsymbol{\Phi}(\mathbf{p})=\mathbf{0}$ nor $\mathbf{C}$ are of use in the sequel, however, and they need not to be formulated at all.

As seen, the formulation of $\mathbf{M}$ and $\mathbf{f}_{g}$ is evident. The formulation of $\mathbf{B}$ is a little more challenging [26]. Let us illustrate this for the case of $\mathbf{B}^{(1)}$ - the first column of $\mathbf{B}$ related to $\sigma_{1}\left(F_{1}\right)$. The force $F_{1}$ is attached to segments $S 1$ (effective origin point $O_{1}$ ) and $S 2$ (insertion point $\left.I_{1}\right)$; see Figs. 7a, b. The inertial frame coordinates of $O_{1}$ and $I_{1}$ are:

$$
\begin{aligned}
x_{O 1} & =x_{S}+\xi_{O 1} \sin \varphi_{1}+\eta_{O 1} \cos \varphi_{1}, \\
y_{O 1} & =y_{S}-\xi_{O 1} \cos \varphi_{1}+\eta_{O 1} \sin \varphi_{1}, \\
x_{I 1} & =x_{S}+l_{1} \sin \varphi_{1}+\xi_{I 1} \sin \varphi_{2}+\eta_{I 1} \cos \varphi_{2}, \\
y_{I 1} & =y_{S}-l_{1} \cos \varphi_{1}-\xi_{I 1} \cos \varphi_{2}+\eta_{I 1} \sin \varphi_{2}
\end{aligned}
$$

where $x_{S}$ and $y_{S}$ are the inertial frame coordinates of the shoulder joint $S, \rho_{O 1}=\left[\xi_{O 1} \eta_{O 1}\right]^{T}$ and $\rho_{I 1}=\left[\begin{array}{ll}\xi_{I 1} & \eta_{I 1}\end{array}\right]^{T}$ are the coordinates of $O_{1}$ and $I_{1}$ in the local coordinate frames $S \xi_{1} \eta_{1}$ and $E \xi_{2} \eta_{2}$, respectively, and $l_{1}$ is the length of $S 1$ (distance between $S$ and $E$ joints). The first column of $\mathbf{B}$, which defines the generalized control force due to $\sigma_{1}, \mathbf{f}_{\sigma 1}=\mathbf{B}^{(1)} \sigma_{1}$, is then

$$
\mathbf{B}^{(1)}=A_{1}\left[\begin{array}{c}
\cos \alpha_{1} \\
-\sin \alpha_{1} \\
\left(y_{C 1}-y_{O 1}\right) \cos \alpha_{1}-\left(x_{O 1}-x_{C 1}\right) \sin \alpha_{1} \\
-\cos \alpha_{1} \\
\sin \alpha_{1} \\
-\left(y_{C 2}-y_{I 1}\right) \cos \alpha_{1}+\left(x_{I 1}-x_{C 2}\right) \sin \alpha_{1}
\end{array}\right]
$$

where the positions $\mathbf{r}_{C 1}=\left[\begin{array}{ll}x_{C 1} & y_{C 1}\end{array}\right]^{T}$ and $\mathbf{r}_{C 2}=\left[\begin{array}{ll}x_{C 2} & y_{C 2}\end{array}\right]^{T}$ of mass centers $C_{1}$ and $C_{2}$, respectively, can be determined from (3) using $\rho_{C 1}=\left[\begin{array}{ll}\xi_{C 1} & \eta_{C 1}\end{array}\right]^{T}$ and $\rho_{C 2}=\left[\begin{array}{ll}\xi_{C 2} & \eta_{C 2}\end{array}\right]^{T}$ 
a)

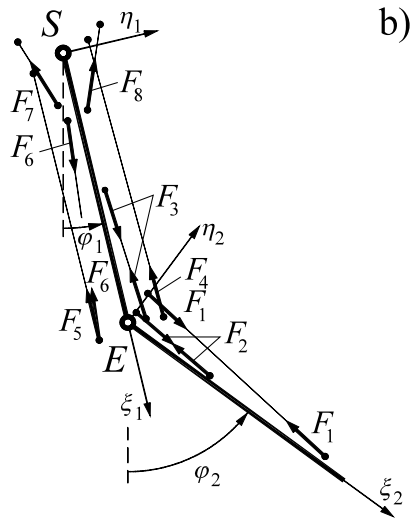

b)

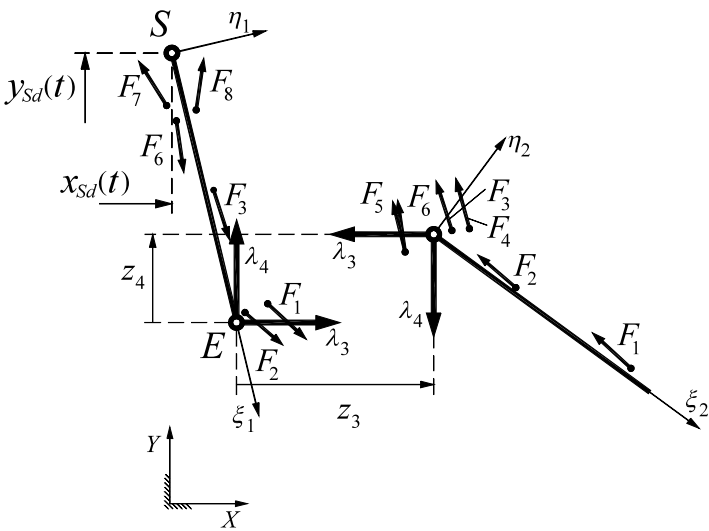

Fig. 7 The muscle forces acting on the segments

instead of $\rho_{O 1}=\left[\begin{array}{ll}\xi_{O 1} & \eta_{O 1}\end{array}\right]^{T}$ and $\rho_{I 1}=\left[\begin{array}{ll}\xi_{I 1} & \eta_{I 1}\end{array}\right]^{T}$, and the sine and cosine of $\alpha_{1}$ (the muscle line inclination angle with respect to the vertical) are:

$$
\sin \alpha_{1}=\frac{x_{I 1}-x_{O 1}}{\sqrt{\left(x_{I 1}-x_{O 1}\right)^{2}+\left(y_{O 1}-y_{I 1}\right)^{2}}} ; \quad \cos \alpha_{1}=\frac{y_{O 1}-y_{I 1}}{\sqrt{\left(x_{I 1}-x_{O 1}\right)^{2}+\left(y_{O 1}-y_{I 1}\right)^{2}}} .
$$

Following this procedure for the other muscles, all $m$ columns of $\mathbf{B}$ can be found, and then

$$
\mathbf{f}_{\sigma}=\sum_{j=1}^{m} \mathbf{f}_{\sigma j}=\sum_{j=1}^{m} \mathbf{B}^{(j)} \sigma_{j}=\mathbf{B} \boldsymbol{\sigma} .
$$

In further derivations the augmented joint coordinate method [30] is applied, which proves especially useful for planar biomechanical models both to obtain the dynamic equations in joint coordinates and to determine the joint reactions [26, 31]. In short, while the traditional joint coordinate scheme $[32,33]$ uses the relationships between the (dependent) absolute coordinates $\mathbf{p}$ and the (independent) joint coordinates $\mathbf{q}$, which are $\mathbf{p}=\mathbf{g}(\mathbf{q})+\boldsymbol{\eta}(t)$ for the case at hand and express the joint constraint equations given explicitly [32], the augmented form of the relationships is

$$
\mathbf{p}=\mathbf{g}(\mathbf{q}, \mathbf{z})+\boldsymbol{\eta}(t)
$$

where $\mathbf{z}$ are the open-constraint coordinates that describe the prohibited relative motions in the joints (Fig. 6b), and the drift in time $\eta(t)$ is induced by attachment of the upper extremity to the moving support $S$, whose motion is specified in time, $\mathbf{r}_{S d}(t)=\left[x_{S d}(t) y_{S d}(t)\right]^{T}$. Actually, since $\mathbf{z}=\mathbf{0},(7)$ is virtually equivalent to $\mathbf{p}=\mathbf{g}(\mathbf{q})+\boldsymbol{\eta}(t)$, and the dependence on $\mathbf{z}$ is introduced only to grasp the prohibited directions related to $\mathbf{z}$, which are also the directions of the respective constraint reactions $\lambda$ [30]. A useful feature of the present formulation is also that the open-constraint coordinates need to be introduced only in those joints in which the reaction forces are to be determined. As an example, let us 'open' only the $E$ joint, and keep 'closed' the connection in the $S$ joint. As such, we involve only $\mathbf{z}^{*}=\left[\begin{array}{ll}z_{3} & z_{4}\end{array}\right]^{T}$, seen in Fig. $7 \mathrm{~b}$, and the related $l^{*}=2$ constraint reactions in the joints are $\lambda^{*}=\left[\begin{array}{ll}\lambda_{3} & \lambda_{4}\end{array}\right]^{T}$. The 
relationship (7) is then

$$
\begin{aligned}
\mathbf{p} & =\left[\begin{array}{c}
x_{C 1} \\
y_{C 1} \\
\theta_{1} \\
x_{C 2} \\
y_{C 2} \\
\theta_{2}
\end{array}\right]=\left[\begin{array}{c}
\xi_{C 1} \sin \varphi_{1}+\eta_{C 1} \cos \varphi_{1} \\
-\xi_{C 1} \cos \varphi_{1}+\eta_{C 1} \sin \varphi_{1} \\
\varphi_{1} \\
l_{1} \sin \varphi_{1}+z_{3}+\xi_{C 2} \sin \varphi_{2}+\eta_{C 2} \cos \varphi_{2} \\
-l_{1} \cos \varphi_{1}+z_{4}-\xi_{C 2} \cos \varphi_{2}+\eta_{C 2} \sin \varphi_{2} \\
\varphi_{2}
\end{array}\right]+\left[\begin{array}{c}
x_{S}(t) \\
y_{S}(t) \\
0 \\
x_{S}(t) \\
y_{S}(t) \\
0
\end{array}\right] \\
& =\mathbf{g}\left(\mathbf{q}, \mathbf{z}^{*}\right)+\boldsymbol{\eta}(t) .
\end{aligned}
$$

The augmented form of the explicit constraint equations (7) allows one to introduce two matrices that are used in the further formulations, i.e.,

$$
\mathbf{D}=\left.\left(\frac{\partial \mathbf{g}}{\partial \mathbf{q}}\right)\right|_{\mathbf{z}=\mathbf{0}} \quad \text { and } \quad \mathbf{E}^{*}=\left.\left(\frac{\partial \mathbf{g}}{\partial \mathbf{z}^{*}}\right)\right|_{\mathbf{z}=\mathbf{0}} .
$$

The $N \times n(6 \times 2)$ matrix $\mathbf{D}$, which arises also from the traditional formulation $\mathbf{p}=\mathbf{g}(\mathbf{q})+$ $\boldsymbol{\eta}(t)$ as $\mathbf{D}=\partial \mathbf{g} / \partial \mathbf{q}$, is an orthogonal complement matrix to the $l \times N(4 \times 6)$ constraint matrix $\mathbf{C}$ introduced in (2), that is $\mathbf{C D}=\mathbf{0} \Leftrightarrow \mathbf{D}^{T} \mathbf{C}^{T}=\mathbf{0}$. By introducing then $\boldsymbol{\gamma}(\mathbf{q}, \dot{\mathbf{q}}, t)=$ $\dot{\mathbf{D}} \dot{\mathbf{q}}+\ddot{\boldsymbol{\eta}}$, which arise from $\ddot{\mathbf{p}}=\mathbf{D} \ddot{\mathbf{q}}+\boldsymbol{\gamma}$, the dynamic equations in joint coordinates $\mathbf{q}$ are produced in the following generic matrix form

$$
\overline{\mathbf{M}}(\mathbf{q}) \ddot{\mathbf{q}}+\overline{\mathbf{d}}(\mathbf{q}, \dot{\mathbf{q}}, t)=\overline{\mathbf{f}}_{g}(\mathbf{q})+\overline{\mathbf{B}}(\mathbf{q}) \mathbf{u}
$$

where $\overline{\mathbf{M}}=\mathbf{D}^{T} \mathbf{M D}$ is the $n \times n(2 \times 2)$ generalized mass matrix related to $\mathbf{q}, \overline{\mathbf{d}}=\mathbf{D}^{T} \mathbf{M} \boldsymbol{\gamma}$ and $\overline{\mathbf{f}}_{g}=\mathbf{D}^{T} \mathbf{f}_{g}$ are the $n$-vectors of generalized forces due to the centrifugal accelerations and gravitational forces, respectively, and $\overline{\mathbf{B}}=\mathbf{D}^{T} \mathbf{B}$ is the $n \times m(2 \times 8)$ matrix of distribution of control inputs $\boldsymbol{\sigma}$ in the directions of $\mathbf{q}$. The $l^{*}=2$ constraint reactions $\lambda^{*}$ in the directions $\mathbf{z}^{*}$ can then be determined from (see $[26,30,31]$ for more details)

$$
\lambda^{*}(\mathbf{q}, \dot{\mathbf{q}}, \ddot{\mathbf{q}}, \boldsymbol{\sigma}, t)=\mathbf{E}^{* T}\left[\mathbf{f}_{g}+\mathbf{B} \boldsymbol{\sigma}-\mathbf{M}(\mathbf{D} \ddot{\mathbf{q}}+\boldsymbol{\gamma})\right]
$$

As seen, what is needed for the derivation of dynamic equations in $\mathbf{q}$, in the symbolic form of (10), are $\mathbf{M}, \mathbf{f}_{g}$, and $\mathbf{B}$ from the absolute coordinate dynamics formulation of (2), and then $\mathbf{D}$ and $\boldsymbol{\gamma}$ arising from $\mathbf{p}=\mathbf{g}(\mathbf{q})+\boldsymbol{\eta}(t)$ as $\mathbf{D}=\partial \mathbf{g} / \partial \mathbf{q}$ and $\boldsymbol{\gamma}=\dot{\mathbf{D}} \dot{\mathbf{q}}+\ddot{\boldsymbol{\eta}}$. The augmented form (7) of the explicit constraint equations, $\mathbf{p}=\mathbf{g}\left(\mathbf{q}, \mathbf{z}^{*}\right)+\boldsymbol{\eta}(t)$, leads to the effective formula (11) for determination of reaction forces in the selected joints (where $\mathbf{z}^{*}$ are introduced). Following the formulation (8) for the studied upper limb, the related matrices $\mathbf{D}$ and $\mathbf{E}^{*}$ are:

$$
\mathbf{D}=\left[\begin{array}{cc}
\xi_{C 1} \cos \varphi_{1}-\eta_{C 1} \sin \varphi_{1} & 0 \\
\xi_{C 1} \sin \varphi_{1}+\eta_{C 1} \cos \varphi_{1} & 0 \\
1 & 0 \\
l_{1} \cos \varphi_{1} & \xi_{C 2} \cos \varphi_{2}-\eta_{C 2} \sin \varphi_{2} \\
l_{1} \sin \varphi_{1} & \xi_{C 2} \sin \varphi_{2}+\eta_{C 2} \cos \varphi_{2} \\
0 & 1
\end{array}\right] ; \quad \mathbf{E}^{*}=\left[\begin{array}{cc}
0 & 0 \\
0 & 0 \\
0 & 0 \\
1 & 0 \\
0 & 1 \\
0 & 0
\end{array}\right]
$$

and the $n$-dimensional vector $\boldsymbol{\gamma}=\dot{\mathbf{D}} \dot{\mathbf{q}}+\ddot{\boldsymbol{\eta}}$ can then easily be obtained after deriving $\dot{\mathbf{D}}$ from $\mathbf{D}$, and introducing $\dddot{\eta}=\left[\begin{array}{llllll}\ddot{x}_{S} & \ddot{y}_{S} & 0 & \ddot{x}_{S} & \ddot{y}_{S} & 0\end{array}\right]^{T}$. 


\subsection{Dynamic equations in natural coordinates}

In this formulation, the human upper extremity model is described by six natural coordinates $\left(x_{i}, y_{i}\right), i=1,2,3$, which are Cartesian coordinates of the basic points seen in Fig. 8 (located at the $S$ and $E$ joints, and at the wrist point $W$ ). As before, the (effective) origin and insertion points of the muscles are defined in the local reference frames attached to the arm $\left(S \xi_{1} \eta_{1}\right)$ and forearm $\left(E \xi_{2} \eta_{2}\right)$. The gravitational and the muscle forces exerted on the segments need then to be distributed between its basic points [34].

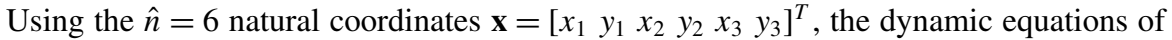
motion for the model can be written in the generic form

$$
\hat{\mathbf{M}} \ddot{\mathbf{x}}=\hat{\mathbf{f}}_{g}+\hat{\mathbf{f}}_{m}-\hat{\mathbf{C}}^{T} \hat{\lambda}
$$

where $\hat{\mathbf{M}}$ is the global mass matrix of the system, $\hat{\mathbf{f}}_{g}$ is the vector of gravitational forces, $\hat{\mathbf{f}}_{m}$ denotes the vector of muscle forces, $\hat{\mathbf{C}}$ is the Jacobian matrix of constraints on $\mathbf{x}, \hat{\boldsymbol{\Phi}}(\mathbf{x}, t)=\mathbf{0}$ and $\hat{\mathbf{C}}=\partial \hat{\boldsymbol{\Phi}} / \partial \mathbf{x}$, and $\hat{\lambda}$ is the vector of associated Lagrange multipliers. The vector of Lagrange multipliers is proportional to the reaction forces associated with the kinematical constraints originating from the constant distance conditions between two successive basic points, and from the rheonomic constraints imposed on the shoulder joint motion, i.e.,

$$
\hat{\boldsymbol{\Phi}}=\left[\begin{array}{c}
\left(x_{2}-x_{1}\right)^{2}+\left(y_{2}-y_{1}\right)^{2}-l_{1}^{2} \\
\left(x_{3}-x_{2}\right)^{2}+\left(y_{3}-y_{2}\right)^{2}-l_{2}^{2} \\
x_{1}-x_{S d}(t) \\
y_{1}-y_{S d}(t)
\end{array}\right]=\mathbf{0}
$$

where $l_{1}$ and $l_{2}$ are the distances between the basic points, and $\mathbf{r}_{S d}(t)=\left[x_{S d}(t) y_{S d}(t)\right]^{T}$ is the description of motion of the shoulder joint which coincides with the first basic point. Note that, in the Lagrange multipliers $\hat{\lambda}=\left[\hat{\lambda}_{1} \hat{\lambda}_{2} \hat{\lambda}_{3} \hat{\lambda}_{4}\right]$ introduced in (13), $\hat{\lambda}_{3}$ and $\hat{\lambda}_{4}$ stand for the horizontal and vertical components of the reaction force in the shoulder joint $S$, and $\hat{\lambda}_{1}$ and $\hat{\lambda}_{2}$ are associated with the internal forces in segments $S 1$ and $S 2$ (note that $\hat{\lambda}_{1}$ and $\hat{\lambda}_{2}$ are not physical forces), respectively, between the $S$ and $E$, and $E$ and $W$ points. The multipliers can then be used to calculate the reaction force in the elbow joint $E$. For a more

Fig. 8 The upper extremity model defined in terms of natural coordinates

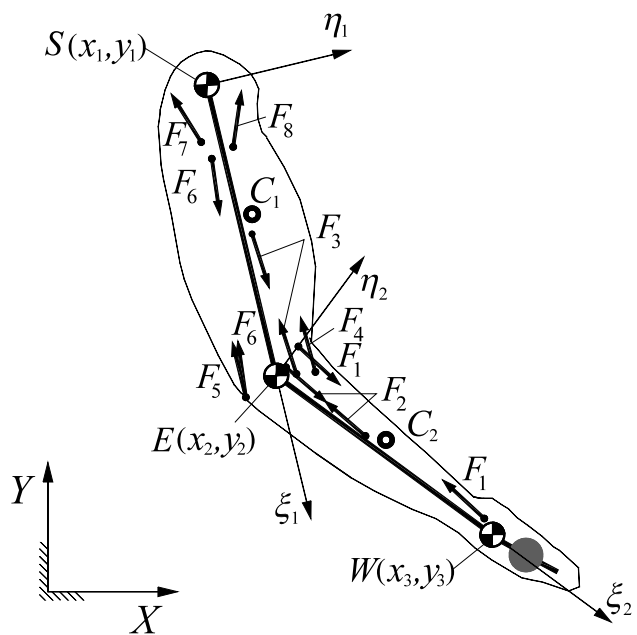


detailed discussion on the problem of determination of joint reactions related to natural coordinate formulations, the reader is referred to, e.g. [5, 34].

The components of (13), expressed in an expanded form, are as follows [34]:

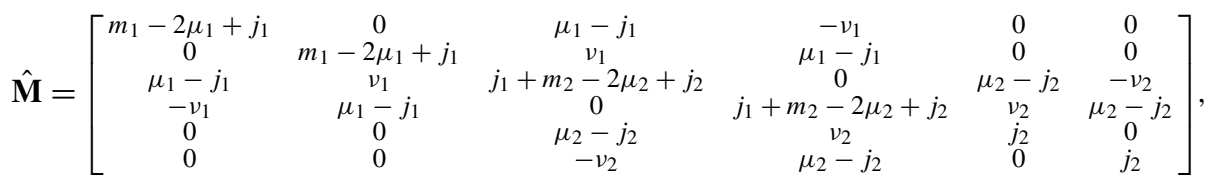

$$
\begin{aligned}
& \hat{\mathbf{f}}_{g}=\left[\begin{array}{c}
v_{1} g \\
-\left(m_{1}-\mu_{1}\right) g \\
-\left(v_{1}-v_{2}\right) g \\
-\left(\mu_{1}-\mu_{2}+m_{2}\right) g \\
-v_{2} g \\
-\mu_{2} g
\end{array}\right] ; \quad \hat{\mathbf{C}}^{T}=\left[\begin{array}{cccc}
2\left(x_{1}-x_{2}\right) & 0 & 1 & 0 \\
2\left(y_{1}-y_{2}\right) & 0 & 0 & 1 \\
2\left(x_{2}-x_{1}\right) & 2\left(x_{2}-x_{3}\right) & 0 & 0 \\
2\left(y_{2}-y_{1}\right) & 2\left(y_{2}-y_{3}\right) & 0 & 0 \\
0 & 2\left(x_{3}-x_{2}\right) & 0 & 0 \\
0 & 2\left(y_{3}-y_{2}\right) & 0 & 0
\end{array}\right]
\end{aligned}
$$

where $\mu_{i}=m_{i} \xi_{C_{i}} / l_{i}, v_{i}=m_{i} \eta_{C_{i}} / l_{i}, j_{i}=I_{i} / l_{i}^{2}$, for $i=1,2$. The individual muscle forces, set as $F_{j}=\sigma_{j} A_{j}(j=1, \ldots, 8)$, are distributed in the following way:

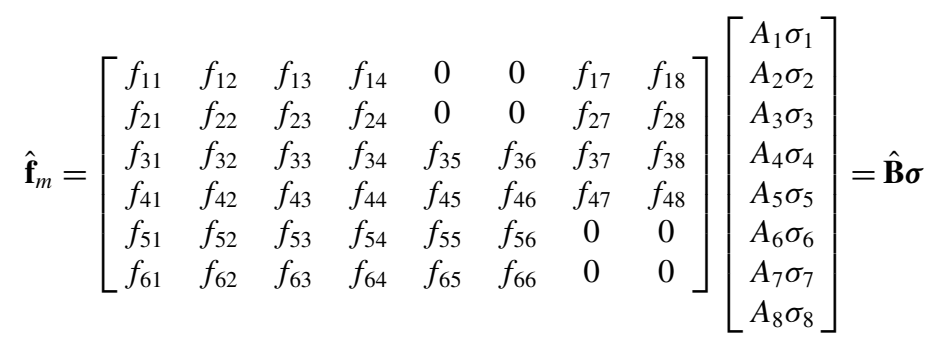

where as before $\sigma=\left[\sigma_{1} \cdots \sigma_{8}\right]^{T}$. The coefficients $f_{i j}(i=1, \ldots, 6, j=1, \ldots, 8)$ which cast the muscle forces into the directions of natural coordinates can be expressed as follows:

$$
\begin{aligned}
f_{1 j} & =\left(1-\frac{\xi_{1}^{j}}{l_{1}}\right) \cos \alpha_{j}-\frac{\eta_{1}^{j}}{l_{1}} \sin \alpha_{j}, \\
f_{2 j} & =\frac{\eta_{1}^{j}}{l_{1}} \cos \alpha_{j}+\left(1-\frac{\xi_{1}^{j}}{l_{1}}\right) \sin \alpha_{j}, \\
f_{3 j} & =\frac{\xi_{1}^{j}}{l_{1}} \cos \alpha_{j}+\frac{\eta_{1}^{j}}{l_{1}} \sin \alpha_{j}+\left(1-\frac{\xi_{2}^{j}}{l_{2}}\right) \cos \alpha_{j}-\frac{\eta_{2}^{j}}{l_{2}} \sin \alpha_{j}, \\
f_{4 j} & =-\frac{\eta_{1}^{j}}{l_{1}} \cos \alpha_{j}+\frac{\xi_{1}^{j}}{l_{1}} \sin \alpha_{j}+\frac{\eta_{2}^{j}}{l_{2}} \cos \alpha_{j}+\left(1-\frac{\xi_{2}^{j}}{l_{2}}\right) \sin \alpha_{j}, \\
f_{5 j}= & \frac{\xi_{2}^{j}}{l_{2}} \cos \alpha_{j}+\frac{\eta_{2}^{j}}{l_{2}} \sin \alpha_{j}, \\
f_{6 j}= & -\frac{\eta_{2}^{j}}{l_{2}} \cos \alpha_{j}+\frac{\xi_{2}^{j}}{l_{2}} \sin \alpha_{j},
\end{aligned}
$$

where $\alpha_{j}$ is the angle between the line of action of force of muscle $j$ and the vertical axis of the global reference frame, the same as those introduced in (5) but defined now with the use 
of $\mathbf{x}$ (which will not be reported here for shortness), and $\left(\xi_{s}^{j}, \eta_{s}^{j}\right)$ are the coordinates (in the local reference frames $S \xi_{1} \eta_{1}$ and $\left.E \xi_{2} \eta_{2}\right)$ of the effective origin/insertion points of muscle $j$ at segment $S 1(s=1)$ or $S 2(s=2)$. The symbolic form of (13) is finally

$$
\hat{\mathbf{M}} \ddot{\mathbf{x}}=\hat{\mathbf{f}}_{g}+\hat{\mathbf{B}}(\mathbf{x}) \mathbf{u}-\hat{\mathbf{C}}^{T}(\mathbf{x}) \hat{\boldsymbol{\lambda}}
$$

\section{Kinematic data used}

The actual performance of flexion-extension movement of the upper limb with a weight $\left(m_{w}=2.5 \mathrm{~kg}\right)$ carried in hand was recorded using a set of digital cameras, and the sampling frequency of measured data was $50 \mathrm{~Hz}$. By tracking the positions of markers placed at the $S, E$ and $W$ points (Fig. 8), we obtained this way first the discrete trajectories $\mathbf{x}_{d}^{*}(t)$ with $K=90$ points for the recorded movement period $t_{K}=1.79 \mathrm{~s}$, and then calculated $\mathbf{q}_{d}^{*}(t)$. The discrete trajectories were then approximated using cubic splines to generate the continuous $\mathbf{q}_{d}(t)$ and $\mathbf{x}_{d}(t)$, respectively, and, on this basis, $\dot{\mathbf{q}}_{d}(t)$ and $\ddot{\mathbf{q}}_{d}(t)\left(\dot{\mathbf{x}}_{d}(t)\right.$ and $\left.\ddot{\mathbf{x}}_{d}(t)\right)$ could be obtained. Smoothness of the characteristics was further improved by applying the procedure once again, that is, using a bigger number $\left(K^{\prime}=360\right)$ of points from the firstfit approximation as input data for the second approximation. In this way, the sampling frequency of 'measured' data was 'improved' to $200 \mathrm{~Hz}$, which is of special importance for improvement of smoothness of $\ddot{\mathbf{q}}_{d}(t)$ and $\ddot{\mathbf{x}}_{d}(t)$, being by assumption linear function between the data points when $\mathbf{q}_{d}^{*}(t)$ and $\mathbf{x}_{d}^{*}(t)$ are approximated by splines. Some of the resulted data used in the inverse dynamics simulation are seen in Fig. 9.

\section{Muscle force estimation}

As defined above, in this study, muscle stresses are considered as controls of the developed upper extremity model, $\sigma=\left[\begin{array}{lll}\sigma_{1} & \cdots & \sigma_{8}\end{array}\right]^{T}, F_{j}=\sigma_{j} A_{j}(j=1, \ldots, 8)$. Due to the control overactuation in musculoskeletal systems, the (redundant) control problem is usually solved using optimization techniques [1-7, 35-38] that apply some predetermined criteria to share the muscular joint torques from the inverse dynamics analysis into the individual muscle efforts. Most often, the redundancy of muscular load sharing is addressed by minimizing a cost (or objective) function appropriately selected for the movement under investigation.

Referred to the dynamics formulation in joint coordinates, (10), the optimization problem can be stated in the following way:

$$
\left\{\begin{array}{l}
\operatorname{minimize} J(\boldsymbol{\sigma}) \\
\text { subject to } \overline{\mathbf{B}}\left(\mathbf{q}_{d}\right) \boldsymbol{\sigma}=\overline{\mathbf{M}}\left(\mathbf{q}_{d}\right) \ddot{\mathbf{q}}_{d}+\overline{\mathbf{d}}\left(\mathbf{q}_{d}, \dot{\mathbf{q}}_{d}, t\right)-\overline{\mathbf{f}}_{g}\left(\mathbf{q}_{d}\right) \\
\quad \text { and } \boldsymbol{\sigma}_{\min } \leq \boldsymbol{\sigma} \leq \boldsymbol{\sigma}_{\max }
\end{array}\right.
$$

where $J$ is a chosen cost (objective) function, $\sigma_{\min }$ and $\sigma_{\max }$ are the physiologically allowable minimal and maximal values of the muscle stresses, and $\mathbf{q}_{d}(t), \dot{\mathbf{q}}_{d}(t)$, and $\ddot{\mathbf{q}}_{d}(t)$ are the measured motion characteristics. In this way, $\sigma_{d}(t)$ are found that minimize the cost function $J(\sigma)$, subject to both the equality constraints $\overline{\mathbf{B}} \boldsymbol{\sigma}=\overline{\mathbf{M}} \ddot{\mathbf{q}}_{d}+\overline{\mathbf{d}}-\overline{\mathbf{f}}_{g}$ given by the equations of motion and the additional boundary condition $\sigma_{\min } \leq \boldsymbol{\sigma} \leq \boldsymbol{\sigma}_{\max }$. When referred 

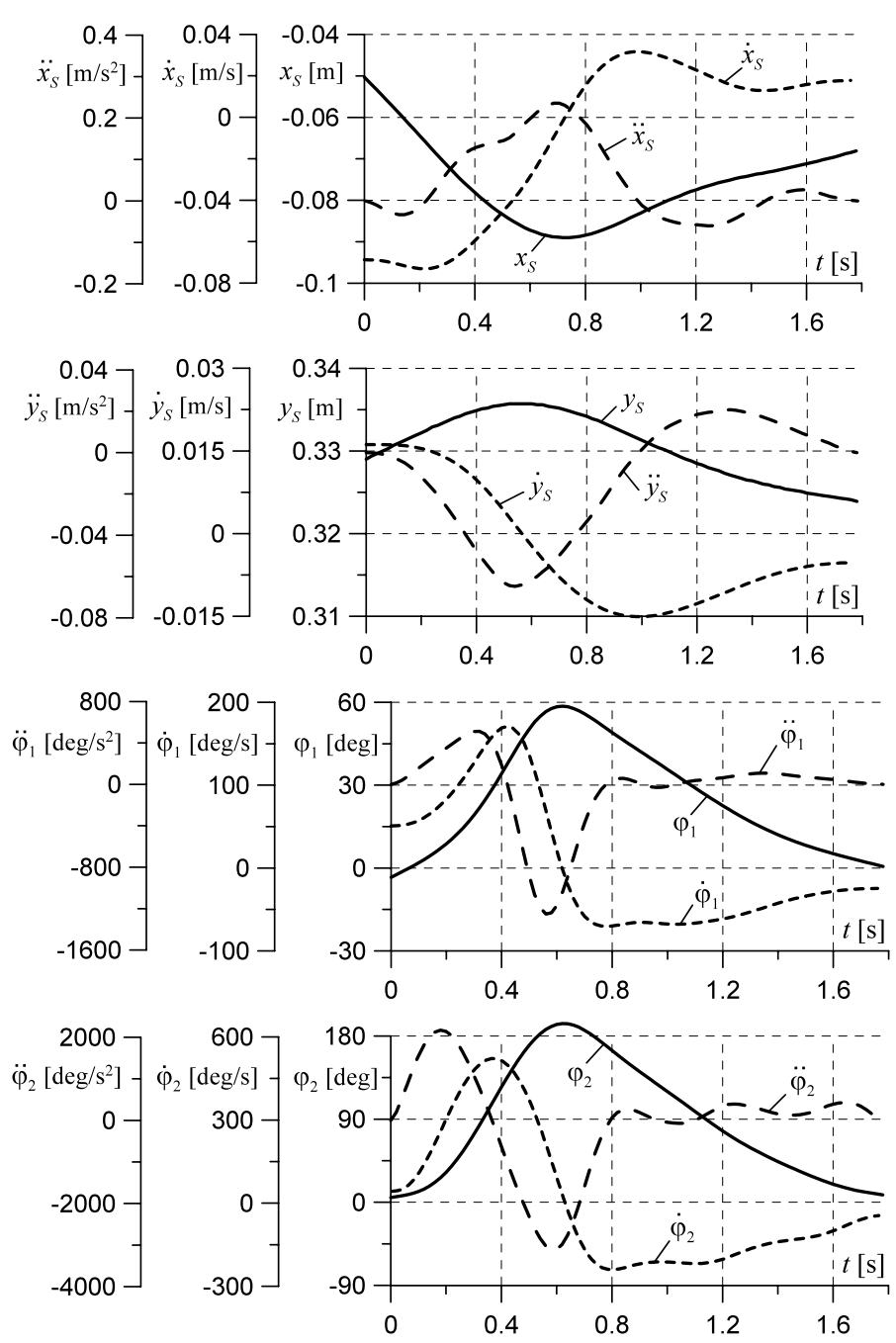

Fig. 9 Examples of motion characteristics used in calculations

to the dynamics formulation in natural coordinates, (19), the optimization problem modifies to

$$
\left\{\begin{array}{l}
\operatorname{minimize} J(\boldsymbol{\sigma}), \\
\text { subject to }\left[\hat{\mathbf{B}}\left(\mathbf{x}_{d}\right):-\mathbf{C}^{T}\left(\mathbf{x}_{d}\right)\right]\left[\begin{array}{l}
\boldsymbol{\sigma} \\
\hat{\lambda}
\end{array}\right]=\hat{\mathbf{M}} \ddot{\mathbf{x}}_{d}-\hat{\mathbf{f}}_{g} \\
\text { and } \boldsymbol{\sigma}_{\min } \leq \boldsymbol{\sigma} \leq \boldsymbol{\sigma}_{\max },
\end{array}\right.
$$

where $\mathbf{x}_{d}(t)$ and $\ddot{\mathbf{x}}_{d}(t)$ are the measured motion characteristics. Here, while only the muscle stresses are optimized to minimize $J(\sigma), \sigma_{d}(t)$ are found together with $\hat{\lambda}_{d}(t)$.

A range of cost functions have been introduced in the literature, followed different (physiologically based) criteria; see, e.g. [4] and [6] for their reviews. For the control problem stated in this contribution, we applied the cost function proposed by Crowninshied and 
Brand [37], which is one of the most frequently employed due to its physiological background related to muscle fatigue,

$$
J=\sum_{j=1}^{m} \sigma_{j}^{p}
$$

and the power values examined in the sequel were $p=1,2,3,4, \ldots, P$. Other alternatives are

$$
J=\sum_{j=1}^{m}\left(\sigma_{j} A_{j}\right)^{p}=\sum_{j=1}^{m} F_{j}^{p}
$$

in which the muscle forces $F_{j}=\sigma_{j} A_{j}$ are directly applied, and

$$
J=\sum_{j=1}^{m}\left(\sigma_{j} A_{j} v_{j}\right)^{p}=\sum_{j=1}^{m}\left(F_{j} v_{j}\right)^{p}=\sum_{j=1}^{m} N_{j}^{p}
$$

where $v_{j}$ is the shortening velocity of muscle $j$, and in which the muscle powers $N_{j}=F_{j} v_{j}$ are applied. With reference to the joint coordinate formulation of (10), we tested also the pseudoinverse method motivated in [1,39], which allows one for a unique mathematical solution for $\sigma_{d}(t)$ from

$$
\sigma_{d}(t)=\overline{\mathbf{B}}^{\dagger}\left(\mathbf{q}_{d}\right)\left[\overline{\mathbf{M}}\left(\mathbf{q}_{d}\right) \ddot{\mathbf{q}}_{d}+\overline{\mathbf{d}}\left(\mathbf{q}_{d}, \dot{\mathbf{q}}_{d}, t\right)-\overline{\mathbf{f}}_{g}\left(\mathbf{q}_{d}\right)\right]
$$

where $\overline{\mathbf{B}}^{\dagger}=\overline{\mathbf{B}}^{T}\left(\overline{\mathbf{B}} \overline{\mathbf{B}}^{T}\right)^{-1}$ is the $m \times n(8 \times 2)$ pseudoinverse (Moore-Penrose generalized inverse [40]) of the rectangular $n \times m(2 \times 8)$ matrix $\overline{\mathbf{B}}$. As proved in [39], the pseudoinverse technique automatically computes the solution which minimizes the quadratic form $(p=2)$ of Crowninshield-Brand function given in (22). Evidently, since both positive and negative muscle forces may be delivered from (25), a post-processing procedure needs then to be applied to keep all the muscle stresses within the boundary constrains $\sigma_{\min } \leq \boldsymbol{\sigma} \leq \sigma_{\max }$ (see, e.g. [26] where the pseudoinverse methodology together with such a post-processing procedure were applied).

\section{Comparative analysis}

\subsection{Joint versus natural coordinates}

There has recently been observed [41] that optimization solutions may depend on the choice of the coordinates used to describe configuration of biomechanical models. For a fivedegree-of-freedom planar musculoskeletal model of human lower extremity, composed of three rigid bodies and actuated by nine Hill-type musculotendon units, a parametric optimization problem was solved using two dynamic formulations: one defined in joint (generalized) coordinates and the other defined in natural coordinates. An advanced muscle model was considered, including its force-length-velocity properties as well as the activation and contraction dynamic characteristics. The parametric optimization problem was then solved for 671 and 854 design variables, respectively, in the generalized and natural coordinate environments. The individual muscle force distribution during raising a leg up occurred to be different for both solutions. Since the searching spaces for optimal solutions differ in dimension, one of the final conclusions in the mentioned paper [41] was 

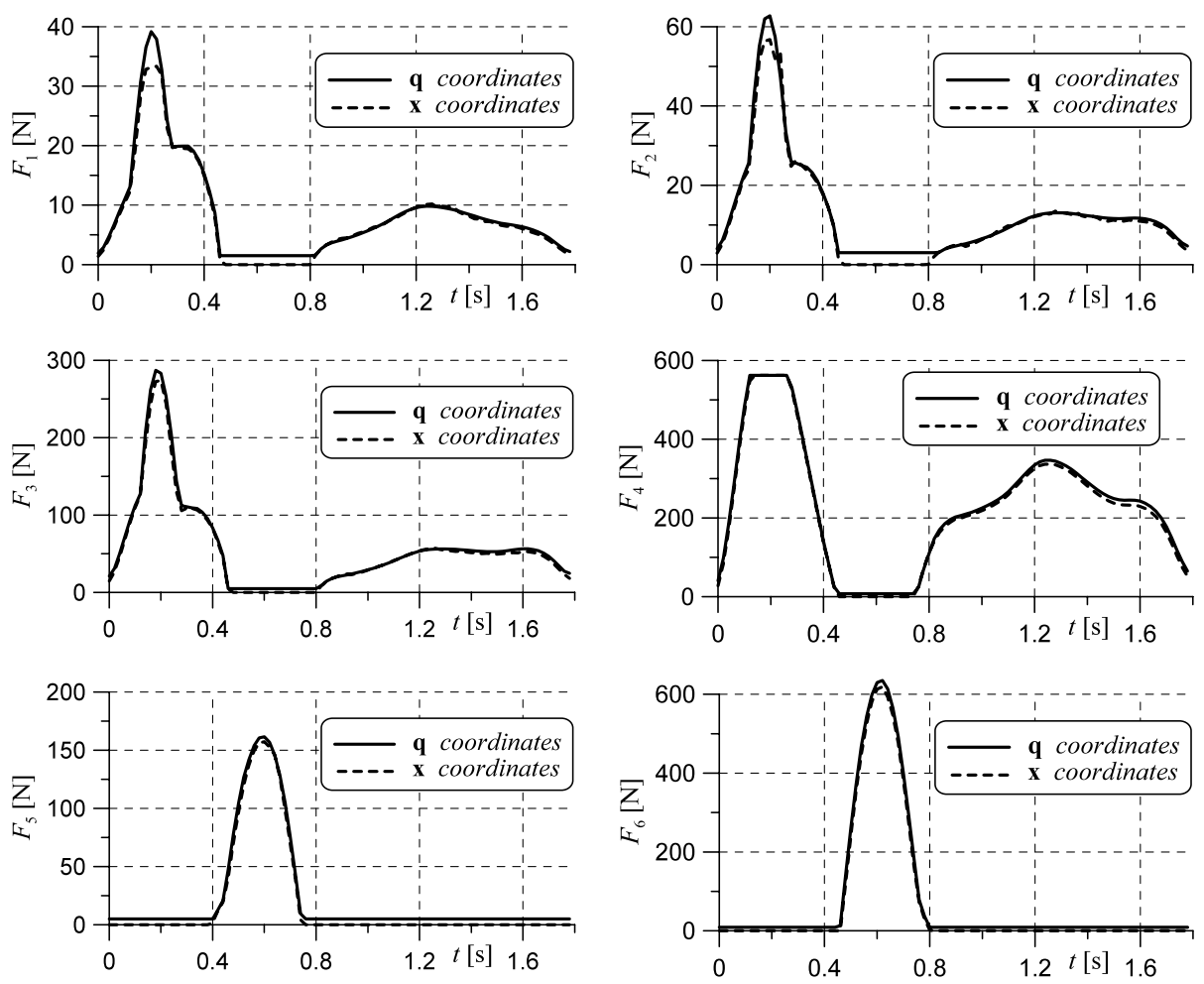

Fig. 10 Variations of muscle forces obtained from formulations in $\mathbf{q}$ and $\mathbf{x}$ coordinate

that the computed differences stem from the numerical reasons. In this study, we intended to verify these observations using the two musculoskeletal models of the upper extremity, defined in joint coordinates $\mathbf{q}$ (Sect. 3.1) and natural coordinates $\mathbf{x}$ (Sect. 3.2), respectively.

For the two models, the optimization procedures applied were as defined in (20) and (21), respectively, for the dynamics formulation in $\mathbf{q}$ and $\mathbf{x}$, and the cost function used in both cases was that of Crowninshied and Brand defined in (22) for $p=2$. The results of the computations are reported in Fig. 10 for muscle forces $F_{1}, \ldots, F_{6}$ (all which exert moments about the elbow joint). As seen, the both optimal solutions match each other quite well. From one point of view, the similar (practically equivalent) optimization results obtained by using different modeling methodologies may be considered as a validation of both approaches and the applied computational codes. On the other hand, the results do not confirm the observation that optimal solutions may depend on the choice of the coordinate system. It must be noted, however, that the upper extremity model used in this study was very simple, and neither the force-length-velocity properties of the muscles nor their activation and contraction dynamics were involved (only 8 and 12 variables were optimized when solving the problem formulated in $\mathbf{q}$ and $\mathbf{x}$ coordinates, respectively). The models used in [41] were much more complex, and much more variables were optimized. The present observations drawn for the very simple model should not thus be generalized. 
Fig. 11 Muscle force estimates for the effective (a) and actual (b) insertion points of muscles $m 5$ and $m 6$ a)

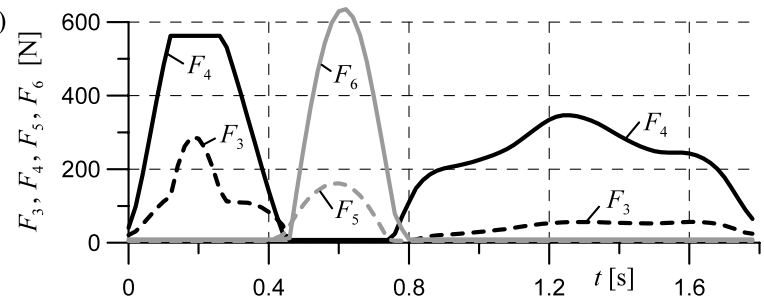

b)

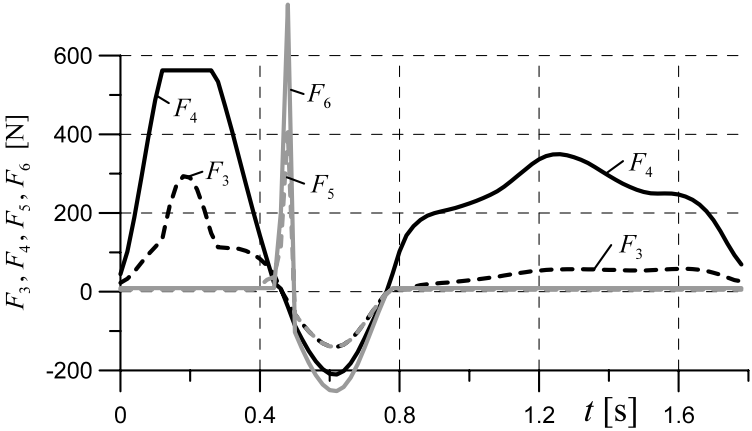

\subsection{Muscle path modeling}

As motivated in Sect. 2, muscle path modeling is of critical importance for reliability of muscle force estimates. This concerns specifically the modeling assumptions used to define the musculotendon paths near the joints, resulted in effective musculotendon path models and their effective origin and/or insertion points, different from the actual ones. In the upper limb model considered in our study, only two muscle attachment points were modeled as fixed in the actual attachment points, that is the origin points of muscles $F_{3}$ and $F_{6}$. As seen in Fig. 4, all the other attachment points are the effective ones, consequent to the modeled muscle moment arms. The simulation results (not reported here for unimportance) proved that the muscle force estimates were practically insensitive to reasonable changes in placement of the origin points of muscles $F_{3}$ and $F_{6}$. The situation was totally different when the muscle moment arms were changed-the muscle force estimates were very sensitive to these changes. A specific analysis of this type is shown in Fig. 11, where the selected muscle force estimates reported previously in Fig. 10, summarized here in graph (a), are confronted to the results obtained assuming the straight musculotendon lines of muscles $m 5$ and $m 6$ as seen in Fig. 2 (with no muscle moment arms assumed in $E$ joint), the latter seen in graph (b). As seen, due to the vanishing moment arms of muscles $m 5$ and $m 6$ they are unable to decelerate the flexion movement in $E$ joint, see Fig. 2, and the optimization procedure leads to non-feasible results (negative muscle forces).

\subsection{Muscle decomposition}

Another type of sensitivity analysis is reported in Fig. 12. Here, we considered the case when muscle $m 4$ (biceps brachii) was divided into two separate (identical) sub-muscles with the same effective attachment points. Now, instead of $m=8$ muscles, we considered the optimization procedure for the model actuated with $m^{\prime}=9$ muscles. As seen from the graphs, the simulation results are substantially different. Firstly, the sum of forces in the submuscles of $m 4$, obtained from the nine-muscle model, is smaller then the force in muscle $m 4$ 

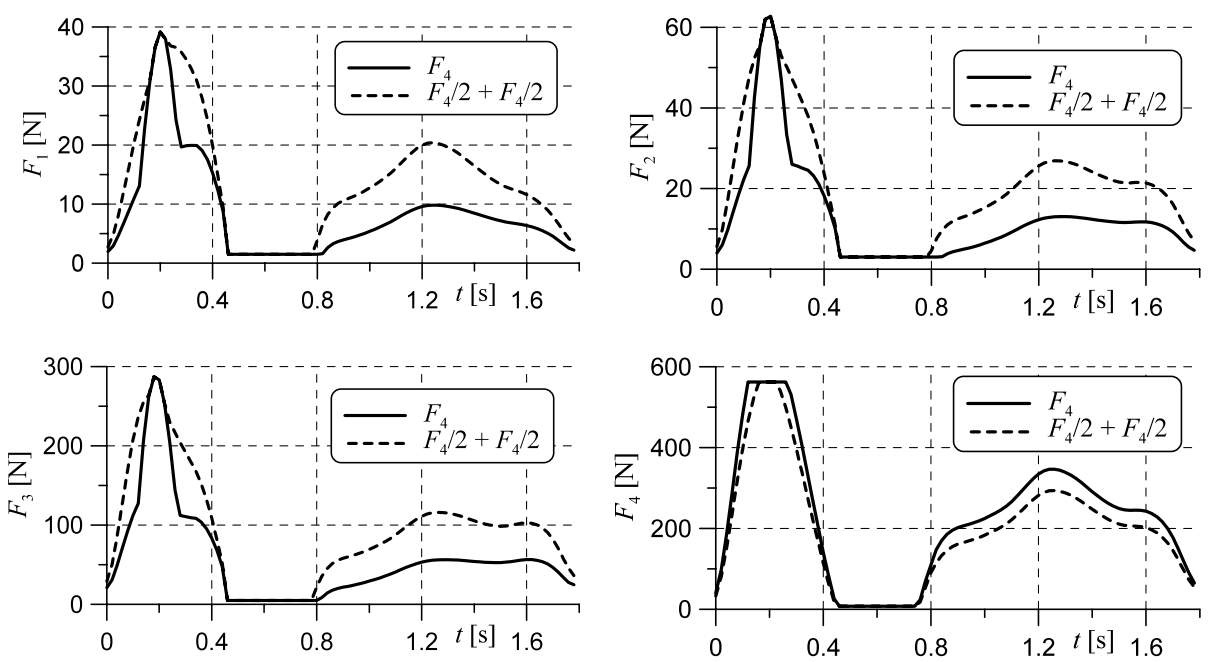

Fig. 12 Selected muscle force estimates for muscle $m 4$ divided into two identical sub-muscles

obtained from the eight muscle model. The result seems evident as squared muscle stresses were minimized according to (22) with $p=2$. Then, in the nine-muscle model, we have two high stresses (which are to be minimized) in the two sub-muscles against one such stress in the eight muscle model. The reduced effort of the two sub-muscles of $m 4$ need then to be compensated by the increased effort of the other muscles who play similar role as muscle $m 4$.

\subsection{Optimization methods}

The last reported example of sensitivity analysis relate the influence of the cost function used in the optimization procedure. In Fig. 13, we reported the results obtained for the cost function of Crowninshied and Brand introduced in (24), for the three power values: $p=1, p=2$ and $p=3$. If $p=1$, the optimization problem is linear, and the muscles with largest product of moment arm and physiological cross-sectional area are recruited first [42]. The courses of muscles $m 3, m 4$ and $m 5$ obtained this way differ from the nonlinear solutions (for $p=2$ and $p=3$ ), in which the other muscles are recruited as well. By contrast, the reaction forces in elbow joint $E$, influenced by the muscle forces, do not change considerably for the three cases. We checked also the other cost functions reported in (23) and (24), in each case observing considerable sensitivity of the results to the cost function chosen. Finally, we tested the pseudoinverse scheme reported in (25) together with the post-processing procedure to keep all the muscle stresses within the boundary constrains $\sigma_{\min } \leq \sigma \leq \sigma_{\max }$. The results were identical to those obtained using the Crowninshied and Brand cost function for $p=2$, which is in accordance with the remarks given in [1].

\section{Conclusion}

In this study, we have shown that the muscle force sharing problem can be very sensitive to the modeling assumptions used in building the musculoskeletal models. Of special importance were the assumptions used to define the musculotendon paths near the joints, resulted 

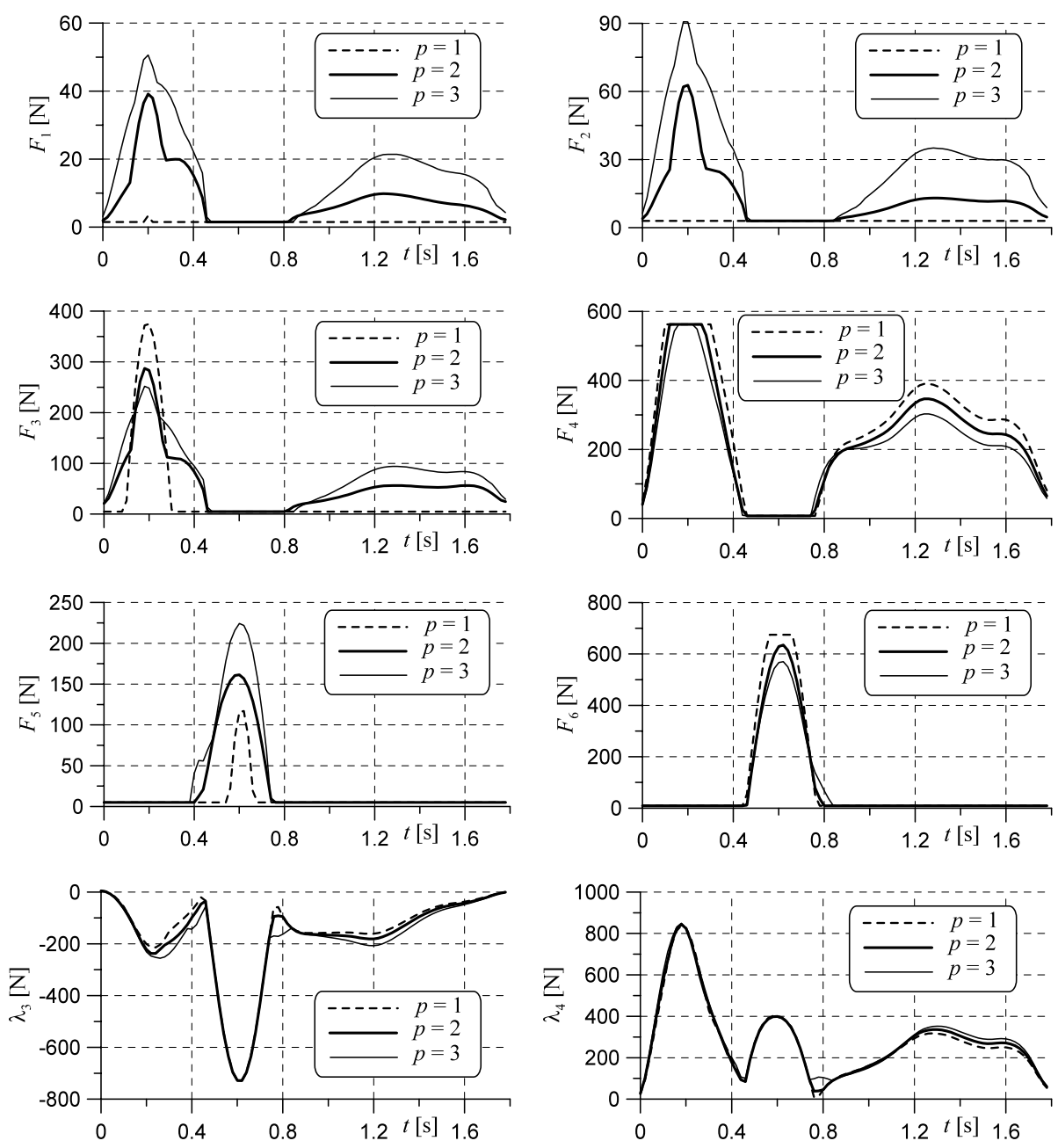

Fig. 13 Estimates of selected muscle forces and joint reactions using different optimization methods

in effective musculotendon path models and their effective origin and/or insertion points, consequent to the non-vanishing muscle moment arms about the actuated joints. The results were also very sensitive to the changes in number of the muscles modeled, obtained for example after decomposing a given muscle into separate sub-muscles. Of great importance was also the choice of the cost function employed in the optimization procedure to distribute the joint torques into the individual muscles. The final conclusion is also that optimization solutions do not depend on the choice of coordinate system, at least for the simple biomechanical model considered in this paper.

Acknowledgements The work was financed in part from the government support of scientific research for years 2010-2012, under grant No. N N501 156438.

Open Access This article is distributed under the terms of the Creative Commons Attribution Noncommercial License which permits any noncommercial use, distribution, and reproduction in any medium, provided the original author(s) and source are credited. 


\section{References}

1. Yamaguchi, G.T.: Dynamic Modeling of Musculoskeletal Motion: a Vectorized Approach for Biomechanical Analysis in Three Dimensions. Dordrecht, Kluwer (2001)

2. Robertson, D.G.E., Caldwell, G.E., Hamill, J., Kamen, G., Whittlesey, S.N.: Research Methods in Biomechanics. Human Kinetics, Champain (2004)

3. Winter, D.A.: Biomechanics and Motor Control of Human Movement. Wiley, New Jersey (2005)

4. Tsirakos, D., Baltzopoulos, V., Bartlett, R.: Inverse optimization: functional and physiological considerations related to the force-sharing problem. Crit. Rev. Biomed. Eng. 25, 371-407 (1997)

5. Silva, M.P.T., Ambrósio, J.A.C.: Human motion analysis using multibody dynamics and optimization tools. Technical Report IDMEC/CPM-2004/001, Lisbon (2004)

6. Erdemir, A., McLean, S., Herzog, W., van den Bogert, A.: Model-based estimation of muscle forces exerted during movements. Clin. Biomech. 22, 131-154 (2007)

7. Ackermann, M., Schiehlen, W.: Physiological methods to solve the force-sharing problem in biomechanics. In: Bottasso, C.L. (ed.) Multibody Dynamics. Computational Methods and Applications, pp. 1-23. Springer, Dordrecht (2008)

8. Seireg, A., Arvikar, R.: Biomechanical Analysis of the Musculoskeletal Structure for Medicine and Sports. Hemisphere Publishing Corporation, New York (1989)

9. Pandy, G.M.: Computer modeling and simulation of human movement. Annu. Rev. Biomed. Eng. 3, 245-273 (2001)

10. Venture, G., Yamane, K., Nakamura, Y.: Identification of human musculo-tendon subject specific dynamics using musculo-skeletal computations and non linear least square. In: Proceedings of the First IEEE/RAS-EMBS International Conference on Biomedical Robotics and Biomechatronics, Pisa, Italy, 20-22 February 2006, pp. 211-216 (2006)

11. Venture, G., Yamane, K., Nakamura, Y.: In-vivo estimation of the human elbow joint dynamics during passive movements based on the musculo-skeletal kinematics computation. In: Proceedings of the 2006 IEEE Conference on Robotics and Automation, Orlando, Florida, 15-19 May 2006, pp. 2960-2965 (2006)

12. Ambrósio, J.A.C., Abrantes, J.M.C.S.: Developments in biomechanics of human motion for health and sports. In: Pereira, M.S. (ed.) A Portrait of State-of-the-Art Research at the Technical University of Lisbon, pp. 531-553. Springer, Dordrecht (2007)

13. Raikowa, R.T., Prilutsky, B.I.: Sensitivity of predicted muscle forces to parameters of the optimizationbased human leg model revealed by analytical and numerical analyses. J. Biomech. 34, 1243-1255 (2001)

14. Strobach, D., Kecskemethy, A., Auer, E., Luther, W., Steinwender, G., Zwick, B.: A sensitivity analysis of origin and insertion points of Hill muscle models with respect to gait dynamics. In: Bottasso, C.L., Masarati, P., Trainelli, L. (eds.) Proceedings of Multibody Dynamics 2007, ECCOMAS Thematic Conference on Advances in Computational Multibody Dynamics, Milano, Italy (2007)

15. Zajac, F.E., Winters, J.M.: Modeling musculoskeletal movement systems: joint and body segmental dynamics, musculoskeletal actuation, and neuromuscular control. In: Winters, J.M., Woo, S.L.-Y. (eds.) Multiple Muscle Systems: Biomechanics and Movement Organizations, pp. 121-148. Springer, New York (1990)

16. Raikova, R.: A model of the flexion-extension motion in the elbow joint-some problems concerning muscle forces modeling and computations. J. Biomech. 29, 763-772 (1996)

17. Brand, R.A., Pedersen, D.R., Friedrich, J.A.: The sensitivity of muscle force predictions to changes in physiologic cross-sectional area. J. Biomech. 19, 589-596 (1986)

18. Winters, J.M., Stark, L.: Muscle models: what is gained and what is lost by varying model complexity. Biol. Cybern. 55, 403-420 (1987)

19. Redl, C., Gfoehler, M., Pandy, M.G.: Sensitivity of muscle force estimates to variations in muscle-tendon properties. Hum. Mov. Sci. 26, 306-319 (2007)

20. Silva, M., Ambrósio, J.: Sensitivity of the results produced by the inverse dynamic analysis of a human stride to perturbed input data. Gait Posture 19, 35-49 (2004)

21. Fidelus, K.: Biomechanical Parameters of Human Upper Limbs. PWN, Warsaw (1971) (in Polish)

22. Yamaguchi, G.T., Sawa, A.G.U., Moran, D.W., Fessler, M.J., Winters, J.M.: A survey of human musculotendon actuator parameters. In: Winters, J.M., Woo, S.L.-Y. (eds.) Multiple Muscle Systems: Biomechanics and Movement Organizations, pp. 717-773. Springer, New York (1990)

23. Zatsiorsky, V.M.: Kinetics of Human Motion. Human Kinetics, Champaign (2002)

24. Eberhard, P., Spägele, T., Gollhofer, A.: Investigations for the dynamical analysis of human motion. Multibody Syst. Dyn. 3, 1-20 (1999)

25. Vukobratović, M., Potkonjak, V., Babković, K., Borovac, B.: Simulation model of general human and humanoid motion. Multibody Syst. Dyn. 17, 71-96 (2007) 
26. Blajer, W., Dziewiecki, K., Mazur, Z.: Multibody modeling of human body for the inverse dynamics analysis of sagittal plane movements. Multibody Syst. Dyn. 18, 217-232 (2007)

27. Celigüeta, J.T.: Multibody simulation of human body motion in sports. In: Proceeding of the XIV International Symposium on Biomechanics in Sports, pp. 81-94. FMH Editions, Madeira (1996)

28. Czaplicki, A., Silva, M.T., Ambrósio, J.C.: Biomechanical modelling for whole body motion using natural coordinates. J. Theor. Appl. Mech. 42, 927-944 (2004)

29. Czaplicki, A.: Are natural coordinates a useful tool in modeling planar biomechanical linkages? J. Biomech. 40, 2307-2312 (2007)

30. Blajer, W.: On the determination of joint reactions in multibody mechanisms. J. Mech. Des. 126, 341350 (2004)

31. Blajer, W., Czaplicki, A.: An alternative scheme for determination of joint reaction forces in human multibody models. J. Theor. Appl. Mech. 43, 813-824 (2005)

32. Schiehlen, W.: Multibody system dynamics: roots and perspectives. Multibody Syst. Dyn. 1, 149-188 (1997)

33. Blajer, W.: A geometric unification of constrained system dynamics. Multibody Syst. Dyn. 1, 3-21 (1997)

34. García de Jalón, J., Bayo, E.: Kinematic and Dynamic Simulation of Multibody Systems: the Real-time Challenge. Springer, New York (1993)

35. Seireg, A., Arvikar, R.J.: The prediction of muscular load sharing and joint forces in the lower extremities during walking. J. Biomech. 8, 89-102 (1975)

36. Patriarco, A.G., Mann, R.W., Simon, S.R., Mansour, J.M.: An evaluation of the approaches of optimization models in the prediction of muscle forces during human gait. J. Biomech. 14, 513-525 (1981)

37. Crowninshield, R.D., Brand, R.A.: A physiologically based criterion of muscle force prediction in locomotion. J. Biomech. 14, 793-801 (1981)

38. Anderson, F.C., Pandy, M.G.: Static and dynamic optimization solutions for gait are practically equivalent. J. Biomech. 34, 153-161 (2001)

39. Yamaguchi, G.T., Moran, D.W., Si, J.: A computationally efficient method for solving the redundant problem in biomechanics. J. Biomech. 28, 999-1005 (1995)

40. Campbell, S.L., Meyer, C.D.: Generalized Inverses of Linear Transformations. Pitman, London (1979)

41. Czaplicki, A.: Optimization solutions depend on the choice of coordinate system. Acta Bioeng. Biomech. 10, 75-79 (2008)

42. Dul, J., Townsend, M.A., Shiavi, R., Johnson, G.E.: Muscular synergism, I: on criteria for load sharing between synergistic muscles. J. Biomech. 17, 663-673 (1984) 